\title{
Analysis of Color Variability of BL Lac during the 1997 and 1999 Outbursts
}

\author{
V. A. Hagen-Thorn, V. M. Larionov, A. V. Hagen-Thorn, S. G. Jorstad \\ \& G. O. Temnov \\ Astronomical Institute, St. Petersburg University, St. Petersburg, \\ Petrodvorets, 2, Bibliotechnaya pl. 198504, Russia
}

\begin{abstract}
The analysis of multicolor observations of BL Lac obtained in the 1997 and 1999 outbursts shows that in both cases the spectral energy distribution of the variable source was unchanged in the course of outburst. The sources have power-law spectra with slightly different spectral indices (the spectrum was flatter in the more powerful outburst of 1997). The variable source is most probably of synchrotron nature.
\end{abstract}

For the solution of the problem of nuclear activity in galaxies, it is very important to know the spectral energy distribution of the central point source responsible for the activity. Unfortunately, this source is not observed in a pure state because of the contribution of the underlying galaxy to the total observed flux. But in some cases, the distribution may be found on the basis of multicolor photometric observations of variability without knowledge of the contribution of the variable source to the total flux.

As we pointed out many times (for details see Hagen-Thorn \& Marchenko 1999), the location of the points corresponding to the fluxes observed in different spectral bands in the "flux-flux diagrams" on straight lines allow the conclusion that the spectral energy distribution of the variable source is constant. The slopes of the straight lines give its relative spectral energy distribution.

The 1997 outburst of BL Lac was the most powerful during the last 20 years. Its multicolor photometric observations were made by several teams. Here we analyze the data published in Webb et al. (1998) and Sobrito et al. (1999). In 1999, another outburst of BL Lac occurred. In this case, we analyze the results of our own observations. To find fluxes from magnitudes, the absolute calibration from Mead et al. (1990) was used.

Figs. 1a,b give "flux-flux diagrams" for both outbursts. It is evident that in both cases the observed points lie on straight lines quite well. Thus we conclude that in both outbursts the spectral energy distribution of the variable source was constant. The slopes of the lines give the observed ratios $\left(F_{i} / F_{R}\right)^{\text {obs }}$, which were then corrected for interstellar reddening using the normal extinction curve.

The logarithms of the ratios $\left(F_{i} / F_{R}\right)^{\text {corr }}$ are compared in Fig. 1c with $\log \nu$ giving the relative spectral energy distributions of the variable sources. Because of the logarithmic scale, we may give an arbitrary vertical shift to one distribution relative to another. The distribution for 1997 is shifted upwards in accordance with the difference in maximum flux in the $\mathrm{R}$ band. 

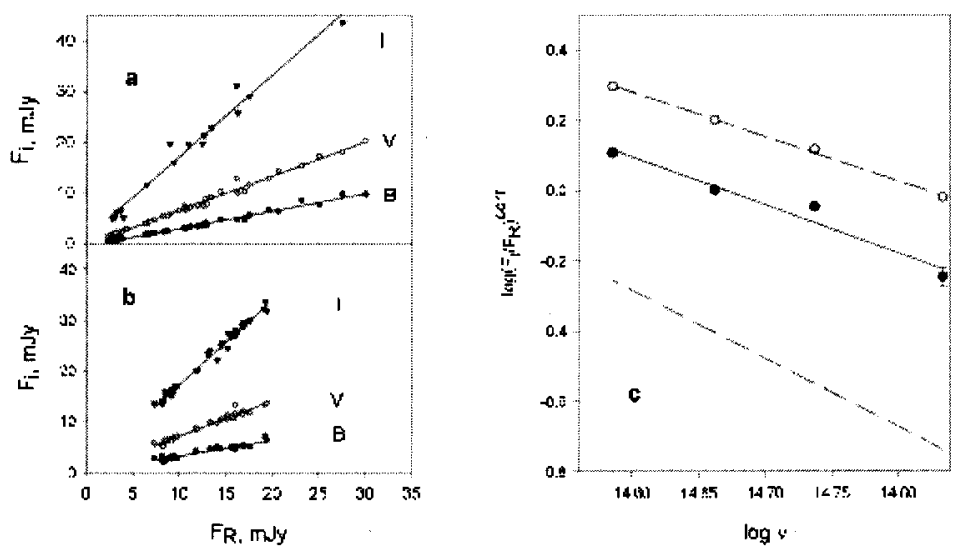

Figure 1. "Flux-flux diagrams" for 1997 (a) and 1999 (b). Spectra of the variable sources (c): filled circles - 1999, open circles - 1997, dashed line - 1983-84.

In both cases the points lie on straight lines showing power-law spectra. Bearing in mind the high observed polarization of BL Lac, one can conclude that the variable sources are of synchrotron nature.

We find the slopes of the lines (spectral indices): $\alpha=-1.29 \pm 0.06$ for 1997 and $\alpha=-1.41 \pm 0.11$ for 1999 . The spectrum appears to be more flat in more powerful outbursts. The tendency is confirmed by the data published in HagenThorn, Marchenko, \& Mikolaichuk (1990). They have found $\alpha=-1.94 \pm 0.07$ for the small outburst of 1983-84. This spectrum is plotted in Fig. 1c by a dashed line. It is shifted downward relative to the 1999 spectrum also in accordance with the flux level in the $R$ band at maximum brightness. For a synchrotron source, such a dependence means that in more powerful outbursts the spectrum of relativistic electrons in the source is harder. Earlier, a similar result was obtained for the blazar OJ 287 (Hagen-Thorn et al. 1998).

Acknowledgments. This work was supported by the Russian Federal Program "Integration" via grants K0232 \& A0007.

\section{References}

Hagen-Thorn, V.A., Marchenko, S.G. 1999, Baltic Astronomy, 8, 575

Webb, J.R., Freedman, I., Howard, E. et al. 1998, AJ, 115, 2244

Sobrito, G., Villata, M., Raiteri, C.M. et al. 1999, Blazar Data, 1, No 5

Mead, A.R.G., Ballard, K.L., Brand, P.W.J.L. et al. 1990, A\&AS, 83, 183

Hagen-Thorn, V.A., Marchenko, S.G., \& Mikolaichuk, O.V. 1990, Astrophisics, 32,244

Hagen-Thorn, V.A., Marchenko, S.G., Takalo, L.O. et al. 1998, A\&AS, 133, 353 